\title{
FINITE GROUPS WHICH CONTAIN A SELF- CENTRALIZING SUBGROUP OF ORDER 3
}

\author{
WALTER FEIT and JOHN G. THOMPSON ${ }^{1)}$
}

Dedicated to Richard Brauer on his sixtieth birthday

\section{$\S 1$. Introduction}

The polyhedral group $(l, m, n)$ is defined in [3] by the presentation

$$
(l, m, n)=\left\langle x, y, z \mid x^{l}=y^{m}=z^{n}=x y z=1\right\rangle .
$$

It is known ([3] page 68) that $(l, m, n)$ is finite if and only if

$$
\frac{1}{l}+\frac{1}{m}+\frac{1}{n}>1 \text {. }
$$

The groups $(2,2, n)$ and $(1, n, n)$ are respectively the dihedral group of order $2 n$ and the cyclic group of order $n$. Using the above mentioned criterion it can be shown that the list of finite polyhedral groups is completed by including

$$
\mathfrak{A}_{4}=(2,3,3), \mathfrak{S}_{4}=(2,3,4) \text { and } \mathfrak{H}_{5}=(2,3,5) \text {. }
$$

Let $G$ be a finite group. If $C_{1}, C_{2}, C_{3}$ are three conjugate classes of $G$ which contain elements of order $l, m, n$ respectively and if $K_{1}, K_{2}, K_{3}$ are the corresponding class sums in the group ring of $G$, a moment's reflection reveals that in order to compute the multiplicity of $K_{3}$ in $K_{1} K_{2}$ by group theoretic methods as distinct from character theoretic methods it is necessary to deal with factor groups of $(l, m, n)$. R. Brauer and K. A. Fowler [1] first realized the importance of this idea for studying finite groups. They were only concerned with the groups $(2,2, n)$ but these were sufficient to prove some powerful results abjut groups of even order. Using the groups $(2,2, n)$ this idea has been used by many authors in recent years and has proved very fruitful for

Received April 12, 1962.

1) The first author was partly supported by A.R.O.D. contract DA-30-115-ORD-976 and the second by the Esso education foundation. Part of this work was dore at the 1960 Summer Conference on Group Theory in Pasadena. 
the study of groups of even order. It is unlikely that knowledge about the other polythedral groups can be utilized as widely as that for the groups (2, $2, n)$. However, the other polyhedral groups can surely play a role in group theory which is not totally eclipsed by the groups $(2,2, n)$.

The purpose of this paper is to illustrate how the above mentioned method can be used with the group $(3,3,3)$. By the result referred to above the group $(3,3,3)$ is infinite. However, it is manageable since, as is shown in section 2 , it has an abelian commutator subgroup.

The following result will be proved in this paper.

TheоRem. Let $G$ be a finite group which contains a self-centralizing subgroup of order 3. Then one of the following statements is true.

(I) $G$ contains a nilpotent normal subgroup $N$ such that $G / N$ is isomorphic to either $\mathfrak{H}_{3}$ or $\bigodot_{3}$.

(II) $G$ contains a normal subgroup $N$ which is a 2-group such that $G / N$ is isomorphic to $\mathfrak{A}_{5}$.

(III) $G$ is isomorphic to PSL $(2,7)$.

As an immediate consequence of this theorem we get

COROLlary. Let $G$ be a non-cyclic simple group which contains a selfcentralizing subgroup of order 3 . Then $G$ is isomorphic to $\mathfrak{A}_{5}$ or PSL $(2,7)$.

If $A$ is a subset of the group $G$ then $C(A), N(A),\langle A\rangle,|A|$ will denote respectively the centralizer of $A$, normalizer of $A$, group generated by $A$ and the number of elements in $A . H \triangleleft G$ means that $H$ is a normal subgroup of $G$. If $p$ is a prime then a $S_{p}$ subgroup of $G$ is a Sylow $p$-subgroup of $G$. Elements of order two are called involutions. For any subgroup $H$ of $G, 1_{H}$ denotes the principal character of $H$. If $\alpha$ is a class function of $H$ then $\alpha^{*}$ denotes the class function of $G$ induced by $\alpha$.

\section{§. The Group $(3,3,3)$.}

Theorem 1. The group $(3,3,3)$ possesses a normal abelian subgroup of index 3.

Proof. Let

$$
(3,3,3)=\left\langle x, y \mid x^{3}=y^{3}=(x y)^{3}=1\right\rangle .
$$

The relation $(x y)^{3}=1$ can be rewritten as 


$$
x y x=y^{-1} x^{-1} y^{-1} \text {. }
$$

Since $y^{-2}=y$ and $x^{-2}=x$ this implies that

$$
x y^{-1} y^{-1} x=y^{-1} x x y^{-1} \text {. }
$$

Hence $x y^{-1}$ and $y^{-1} x$ commute. Conjugating this relation by $x$ and $x^{2}$ yields

$$
\begin{aligned}
& y^{-1} x \cdot x^{-1} y^{-1} x^{-1}=x^{-1} y^{-1} x^{-1} \cdot y^{-1} x \\
& x^{-1} y^{-1} x^{-1} \cdot x y^{-1}=x y^{-1} \cdot x^{-1} y^{-1} x^{-1} .
\end{aligned}
$$

Thus $H=\left\langle x y^{-1}, y^{-1} x, x^{-1} y^{-1} x^{-1}\right\rangle$ is abelian. Since $x$ permutes the three elements $x y^{-1}, y^{-1} x, x^{-1} y^{-1} x^{-1}$ cyclically $x$ normalizes $H$. Hence $y$ also normalizes $H$ as $x y^{-1} \in H$. Thus $H$ is a normal subgroup of $(3,3,3)$. Since $(3,3,3)$ can be mapped homomorphically onto a non abelian group of order 27, $H$ is a proper subgroup. As $x y^{-1} \in H$ and $x \notin H, H$ has index 3 as required.

\section{§3. Proof of the Theorem}

Throughout this section let $G$ be a counter-example of minimum order to the theorem stated in section 1 . Let $x$ be an element of $G$ such that $x^{3}=1$ and $C(x)=\langle x\rangle$. It is easily seen that $\langle x\rangle$ is a $S_{3}$ subgroup of $G$. We will eventually derive a contradiction from the assumed existence of $G$. This will be done in a series of Lemmas.

\section{LEMMA 1. $G$ is a non-cyclic simple group.}

Proof. Suppose this is not the case and let $H$ be a minimal normal subgroup of $G$. Suppose that 3 divides $|H|$. Then the Sylow theorems imply that $G=N(\langle x\rangle) H$. Thus $[G: H]=2$ and $N(\langle x\rangle) \cap H=\langle x\rangle$. Hence by Burnside's transfer theorem $H$ contains a normal 3-complement $H_{0}$. Thus $H_{0} \triangleleft G$ and the minimality of $H$ implies that $H_{0}=1$. Consequently $G$ is isomorphic to $\bigodot_{3}$ contrary to assumption.

Assume now that 3 divides $[G: H]$. Then $\langle x\rangle H$ is a Frobenius group. Thus $H$ is nilpotent ([2], page 91 ). It is easily seen that $G / H$ satisfies the hypotheses of the theorem stated in section 1 . Thus by induction $G / H$ satisfies condition (I), (II), or (III). Therefore $G$ contains a normal subgroup $N$ such that $G / N$ is isomorphic to $\mathfrak{U}_{3}, \mathfrak{\subseteq}_{3}, \mathfrak{I}_{5}$ or $\operatorname{PSL}(2,7)$. In any case $\langle x\rangle N$ is a Frobenius group and $N$ is nilpotent ([2], page 91 ). If $G / N$ is isomorphic to $\mathfrak{A}_{3}$ or $\mathfrak{S}_{3}$ nothing remains to be proved. 
Let $p$ be a prime dividing $|N|$. We will show that $p=2$ if $G / N$ is isomorphic to $\mathfrak{H}_{5}$ while $|N|=1$ if $G / N$ is isomorphic to $\operatorname{PSL}(2,7)$. By induction it may be assumed that $N$ is an elementary abelian $p$-group. Suppose that $A$ is a subgroup of $G$ such that $A \cap N=1, A \neq A^{\prime},\left[A: A^{\prime}\right]=3$ and $p$ does not divide $|A|$. Then $A$ is a Frobenius group acting on $N$. Since $x$ has no fixed points on $N$ we get that $A^{\prime}$ acts trivially on $N$. Thus $N \subset C(N)$. Since $C(N) \triangleleft G$ and ${ }^{\prime} \mathfrak{H}_{5}, P S L(2,7)$ are simple, this yields that $C(N)=G$. Thus $N \subseteq C(x)$ or $N=1$. As both $\mathfrak{H}_{5}$ and $P S L(2,7)$ contain a subgroup $A$ which is isomorphic to $\mathfrak{A}_{4}$ this implies that $p=2$. As $\operatorname{PSL}(2,7)$ contains a nonabelian subgroup of order 21 we get that $N=1$ in this case. The proof is complete in all cases.

Lemma 2. G contains only one conjugate class of elements of order three, and $|G|$ is even.

Proof. Lemma 1 and Burnside's transfer theorem imply that $N(\langle x\rangle) \neq\langle x\rangle$. Thus $|N(\langle x\rangle)|=6$. The result is immediate.

Lemma 3. There exist exactly two non-principal irreducible characters $\theta, \chi$ of $G$ which do not vanish on $x$. They can be chosen so that $\theta(x)=1, \chi(x)=-1$ and $1+\theta(y)-\chi(y)=0$ for $y$ not conjugate to $x$.

Proof. Let $\lambda$ be a nonprincipal irreducible character of $\langle x\rangle$. Let $\alpha$ be the generalized character of $N(\langle x\rangle)$ induced by $1 ;, \cdots$, Then it is easily seen that $\left\|\alpha^{*}\right\|^{2}=3$ and

$$
\begin{array}{ll}
\alpha^{*}(x)=\alpha(x)=3 & \text { for } y \text { not conjugate to } x .
\end{array}
$$

Consequently $\alpha^{*}=1_{G}+\theta-\chi$, where $\%, \theta$ are distinct nonprincipal irreducible characters of $G$. Furthermore $1+\theta(y)-\%(y)=0$ for $y$ non conjugate to $x$. Furthermore by (1)

$$
1=\left(\theta, \alpha^{*}\right)=\frac{1}{6} 3\left\{\theta\left(x^{-1}\right)+\theta(x)\right\}=\theta(x)
$$

Thus by (1) $\chi(x)=-1$. Consequently

$$
|C(x)|=3=1+|\theta(x)|^{2}+|\%(x)|^{2} .
$$

Hence the orthogonality relations imply that every irreducible character of $G$. 
distinct from $1_{G}, \%$ and $\theta$ vanishes on $x$. The proof is complete.

The next lemma is due to R. Brauer and M. Suzuki. We are indebted to them for informing us of the result.

Lemma 4. G contains exactly one class of involutions.

Proof. Any two involutions which normalize a subgroup of $G$ of order 3 are conjugate. Suppose that $G$ contains two classes of involutions then there is one class containing involutions such that $u v$ is not conjugate to $x$ for any $u, v$ in that class. Let $C$ be the group algebra sum of this class of involutions and let $K$ be the group algebra sum of the elements of order 3 . Thus the coefficient of $K$ in $C^{2}$ is zero. Hence by a well-known formula ([2], page 316)

$$
\left.\frac{|G|}{|C(u)|^{2}} \mid \sum \frac{\zeta_{i}(u)^{2} \overline{\zeta_{i}(x)}}{\zeta_{i}(1)}\right]=0
$$

where $\zeta_{i}$ ranges over all the irreducible characters of $G$. In view of Lemma 3 this implies that

$$
1+\frac{\theta(u)^{2}}{\theta(1)}-\frac{\{\theta(u)+1\}^{2}}{\theta(1)+1}=0
$$

Therefore

$$
H(1)^{2}+\theta(1)+\theta(1) \theta(u)^{2}+\theta(u)^{2}-\theta(1) \theta(u)^{2}-2 \theta(1) \theta(u)-\theta(1)=0,
$$

or equivalently

$$
\theta(1)^{2}-2 \theta(1) \theta(u)+\theta(u)^{2}=0 .
$$

Thus $\{\theta(1)-\theta(u)\}^{2}=0$ and $\theta(1)=\theta(u)$. This implies that $u$ lies in a proper normal subgroup of $G$ contrary to Lemma 1 . The proof is complete.

Throughout the rest of this paper the following notation will be used.

$K$ is the group algebra sum of all elements of order 3 in $G$.

$C$ is the group algebra sum of all involutions in $G$.

$u$ is a fixed involution in $G$.

$M_{1}, \ldots, M_{s+m}$ is a complete set of representatives of the conjugate classes of maximal solvable subgroups of $G$ whose order is divisible by 3. By induction each $M_{i}$ contains a normal nilpotent subgroup $N_{i}$. The notation is chosen so that

$$
M_{i} / N_{i} \text { is isomorphic to } \mathfrak{T}_{3} \quad \text { for } 1 \leq i \leq k
$$




$$
M_{i} / N_{t} \text { is isomorphic to } \bigodot_{3} \quad \text { for } k<i \leq s+m
$$

where $\left|N_{i}\right|$ is odd for $k+1 \leq i \leq s$ and $\left|N_{i}\right|$ is even for $s+1 \leq i \leq s+m$.

Let $N_{i}=H_{i} \times T_{i}$, where $\left|H_{i}\right|$ is odd and $T_{i}$ is a 2-group. Define

$$
h_{i}=\left|H_{i}\right|, t_{i}=\left|T_{i}\right| \quad \text { for } 1 \leq i \leq s+m .
$$

Lemma 5. $H_{i}$ is a Hall subgroup of $G$ for $1 \leq i \leq s+m$ and $\left(h_{i}, h_{j}\right)=1$ for $1 \leq i<j \leq s+m$. $N_{i}$ is a Hall subgroup of $G$ for $1 \leq i \leq k$ and $\left(\left|N_{i}\right|,\left|N_{j}\right|\right)=1$ for $1 \leq i<j \leq k$.

Proof. Let $P$ be a $S_{p}$ subgroup of $N_{i}$ for some prime $p$. Lemma 1 and the maximality of $M_{i}$ imply by induction that $N(P)=M_{i}$ if $p>2$ or if $1 \leq i \leq k$. Thus in these cases $P$ is a $S_{p}$ subgroup of $G$. Hence $H_{i}, N_{i}$ are Hall subgroups for $1 \leq i \leq s+m, 1 \leq i \leq k$ respectively. If one of the other statements of the Lemma is false it may be assumed by taking conjugates that for some $S_{p}$ subgroup $P$ of $G, P \subseteq H_{i} \cap H_{j}, i \neq j$, or $P \subseteq N_{i} \cap N_{j}$ and $1 \leq i<j \leq k$. Hence in either case $\left\langle M_{i}, M_{j}\right\rangle \subseteq N(P)$. By the first part of the lemma this implies that $M_{i}=M_{j}$ contrary to the definition of the groups $M_{i}$.

Lemma 5 yields that

$$
g=|G|=3 \cdot 2^{n} g_{0} \prod_{i=1}^{s+m} h_{i}, \quad\left(g_{0}, 6\right)=1
$$

Furthermore $t_{i} \neq 1$ for at most one value of $i$ with $1 \leq i \leq k$. Choose the notation so that

$$
\begin{aligned}
& t_{1}=1 \text { or } t_{1}=2^{n} \\
& t_{i}=1 \text { for } 2 \leq i \leq k \\
& t_{i} \neq 1 \text { for } s+1 \leq i \leq s+m . \\
& h_{s+1} \geq h_{i} \quad \text { for } s+1 \leq i \leq s+m .
\end{aligned}
$$

LEMma 6.

$$
\frac{g}{9}<\frac{g}{9}\left\{1+\frac{1}{\theta(1)}-\frac{1}{\theta(1)+1}\right\} \leq 1+2 \sum_{i=1}^{k}\left(h_{i} t_{i}-1\right)+\sum_{i=k+1}^{s+m}\left(h_{i} t_{i}-1\right)
$$

Proof. The first inequality is trivial. By Lemma 3 the second term in (5) is the multiplicity of $K$ in $K^{2}$. Thus the second term in (5) is the number of ordered pairs $(y, z)$ with $y z=x$ and $y, z$ of order 3 . Since $\langle y, z\rangle$ is a homomorphic image of $(3,3,3)$ it is solvable by Theorem 1 . Thus for every such pair, $\langle y, z\rangle$ is contained in a conjugate of some $M_{i}, 1 \leq i \leq s+m$. 
Suppose that $x \in M_{i} \cap w^{-1} M_{i} w$ for some $w \in G$. Then $w x w^{-1} \in M_{i}$. There exists $w_{1} \in M_{i}$ such that $w_{1}\langle x\rangle w_{1}^{-1}=w\langle x\rangle w^{-1}$. Hence it may be assumed that $w \in N(\langle x\rangle)$. This implies that $x$ is contained in exactly one conjugate of $M_{i}$ for $k+1 \leq i \leq s+m$ and $x$ is contained in exactly two conjugates of $M_{i}$ for $1 \leq i \leq k$. The number of ordered pairs $(y, z)$ with $y z=x, y, z$ of order 3 and $y, z \in M_{i}$ is easily seen to be $h_{i} t_{i}$. If the pair $\left(x^{2}, x^{2}\right)$ is counted just once the second inequality in (5) follows.

Lemma 7. Let $a$ be the multiplicity of $C$ in $K^{2}$. Then

$$
a \geq \sum_{i=s+1}^{s+m} \frac{|C(u)|}{2 h_{i} t_{i}} h_{i} t_{i}
$$

Proof. Let $(y, z)$ be an ordered pair of elements of order 3 such that $y z=u$. Then $\langle y, z\rangle$ is isomorphic to $(3,3,2)=\mathfrak{A}_{4}$.

Suppose that $\langle y, z\rangle$ is contained in two distinct subgroups which are respectively conjugate to $M_{i}$ and $M_{j}$ with $s+1 \leq i<j \leq s+m$. By changing notation it may be assumed that $\langle y, z\rangle \subseteq M_{i} \cap M_{j}$, where $M_{i} \cap M_{j}$ is maximal among all such intersections. Let $D=N_{i} \cap N_{j}$, then $N(\langle y\rangle) \subseteq N(D)$. Since $\left[\langle y, z\rangle:\langle y, z\rangle^{\prime}\right]=3$ it follows that $\langle y, z\rangle^{\prime} \subseteq D$. Define

$$
L_{i}=N(D) \cap N_{i}, \quad L_{j}=N(D) \cap N_{j} .
$$

Then $\left\langle L_{i}, L_{j}\right\rangle \subseteq N(D)$. Thus by Lemma $1\left\langle L_{i}, L_{j}\right\rangle \neq G$. Furthermore

$$
N(\langle y\rangle) \subseteq N\left(L_{i}\right) \cap N\left(L_{j}\right) \subseteq N\left(\left\langle L_{i}, L_{j}\right\rangle\right) .
$$

Let $M$ be a maximal solvable subgroup of $G$ which contains $N(\langle y\rangle)\left\langle L_{i}, L_{j}\right\rangle$ and let $N$ be the maximal normal nilpotent subgroup of $M$. By induction $M / N$ is isomorphic to $\bigodot_{3}$. Since $N(\langle y\rangle) \cap L_{i}=\langle 1\rangle$ this implies that $L_{i} \subseteq N$. Since $N_{i} \nsubseteq N_{j}$ we have that $D \neq N_{i}$. Thus $D \neq L_{i}$ as $N_{i}$ is nilpotent. Therefore $M_{i} \cap M_{j} \subset M_{i} \cap M$. A similar argument shows that $M_{i} \cap M_{j} \subset M_{j} \cap M$. As $M$ cannot be conjugate to both $M_{i}$ and $M_{j}$ one of these inclusions contradicts the maximal nature of $M_{i} \cap M_{j}$. Thus $\langle y, z\rangle$ is not contained in two subgroups which are conjugate $M_{i}, M_{j}$ respectively with $s+1 \leq i<j \leq s+m$.

If $\langle y, z\rangle \subseteq M_{i} \cap w^{-1} M_{i} w$ for $w \in G$ then $N(\langle y\rangle) \subseteq M_{i} \cap w^{-1} M_{i} w$. This implies that $M_{i}=w^{-1} M_{i} w$. Let $u$ lie in exactly $m_{i}$ subgroups conjugate to $N_{i}$. Since $M_{i}$ contains at least $h_{i} t_{i}$ ordered pairs $(y, z)$ with $y^{3}=z^{3}=1, y z=u$, this implies that

$$
a \geq \sum_{i=s+1}^{s+m} h_{i} t_{i} m_{i}
$$


Clearly $m_{i} \geq\left[C(u): C(u) \cap M_{i}\right] \geq \frac{|C(u)|}{2 h_{i} t_{i}}$. The lemma follows.

LEMMA 8.

$$
\sum_{i=s+1}^{s+m} \frac{|C(u)|}{2 h_{i} t_{i}} h_{i} t_{i} \leq \frac{g}{3}
$$

Proof. Let a be the multiplicity of $C$ in $K^{2}$. Then by Lemma 3

$$
a=\frac{g}{9}\left\{1+\frac{\theta(u)}{\theta(1)}+\frac{\chi(u)}{\chi(1)}\right\} \leq \frac{g}{3} .
$$

The result now follows from Lemma 7 .

LeMma 9. $|C(u)|=2^{n} h$ with $h \neq 1$.

Proof. By Lemma $4, u$ is in the center of a $S_{2}$-subgroup of $G$. Suppose that $h=1$. Then ([4], p. 870, [5], Theorem 3) $G$ is isomorphic to $P S L(2,9), P S L$ $(3,4)$ or $P S L(2, q)$ for $g$ a prime or a power of 2 . Since 9 does not divide $g$ the first two possibilities cannot occur. If $q$ is odd $\operatorname{PSL}(2, q)$ contains cyclic subgroups of order $\frac{q+1}{2}$ and $\frac{q-1}{2}$. Thus one of $p, \frac{p-1}{2}, \frac{p+1}{2}$ equals 3 . Hence $p=3,5,7$. Since $P S L(2,3), P S L(2,5)$ are respectively isomorphic to $\mathfrak{A}_{4}$, $\mathfrak{H}_{5}$ these possibilities cannot occur. If $q$ is a power of 2 , then $q \pm 1=3$ and so $q=2$ or 4 . As $P S(2,2)$ is not simple and $\operatorname{PSL}(2,4)$ is isomorphic to $\mathfrak{A}_{5}$ we get that $h \neq 1$.

The proof of the main Theorem is now divided into three cases.

$$
\begin{array}{ll}
\text { Case I. } & h=h_{s+1}, t_{1} \neq 2^{n} \\
\text { Case II. } & h=h_{s+1}, t_{1}=2^{n} \\
\text { Case III. } & h \neq h_{s+1} .
\end{array}
$$

In cases I and II $h_{i}=1$ for $i>s+1$. In case II $h_{1}=1$. Thus in cases I and II Lemmas 6, 7 and 8 and equation (2) yield that

$$
\frac{2^{n} g_{0} \prod_{i=1}^{s+m} h_{i}}{3} \leq 2\left\{h_{1} t_{1}+\sum_{i=2}^{k} h_{i}\right\}+h t_{s+1}+\frac{1}{h}\left\{2^{n} g_{0} \prod_{i=1}^{s+m} h_{i}\right\} .
$$

Since $(h, 6)=1$ and $h \neq 1$ by Lemma 9 we get that $h \geq 5$. Thus in cases I or II we get

$$
\frac{2^{n} g_{0} \prod_{i=1}^{s+m} h_{i}}{15} \leq \frac{1}{2}\left(\frac{1}{3}-\frac{1}{h}\right) 2^{n} g_{0} \prod_{i=1}^{s+m} h_{i} \leq h_{1} t_{1}+\sum_{i=2}^{k} h_{i}+h_{i_{s+1}}
$$


Hence in Case I we get

$$
\frac{2^{n} g_{0} \prod_{i=1}^{s+m} h_{i}}{15} \leq \frac{1}{2}\left(\frac{1}{3}-\frac{1}{h}\right) 2^{n} g_{0} \prod_{i=1}^{s+m} h_{i} \leq \sum_{i=1}^{k} h_{i}+h 2^{n} .
$$

Since $t_{s+1} \leq 2^{n-1}$ we get in case II that

$$
h_{1} t_{1}+h t_{s+1} \leq 2^{n}+2^{n-1} h<2^{n} h .
$$

Thus in case II

$$
\frac{2^{n} g_{0} \prod_{i=1}^{s+m} h_{i}}{15} \leq \frac{1}{2}\left(\frac{1}{3}-\frac{1}{h}\right) 2^{n} g_{0} \prod_{i=1}^{s+m} h_{i} \leq \sum_{i=2}^{k} h_{i}+h 2^{n} .
$$

In case III let $h_{0}$ be the minimum value of $h / h_{i}$ for $s+1 \leq i \leq s+m$. Hence $h_{0} \geq 5$. Thus

$$
\frac{2^{n} g \prod_{i=1}^{s+m} h_{i}}{3} \leq 2\left\{h_{1} t_{1}+\sum_{i=2}^{k} h_{i}\right\}+\frac{2^{n} g_{0} \prod_{i=1}^{s+m} h_{i}}{h_{0}}
$$

or in case III

$$
2^{n} g_{0} \prod_{i=1}^{s+m} h_{i} \leq \frac{1}{15}\left(\frac{1}{3}-\frac{1}{h_{0}}\right) 2^{n} g_{0} \prod_{i=1}^{s+m} h_{i} \leq h_{1} t_{1}+\sum_{i=2}^{k} h_{i} .
$$

For convenience the following notation is now introduced.

Case I $q=k+1, z=h$

$$
\left\{x_{1}, \ldots, x_{q}\right\} \text { is the set }\left\{h_{1}, \ldots, h_{k}, h 2^{n}\right\}
$$

in ascending order, and

$$
v=\frac{1}{h} g_{0} \prod_{i=k+1}^{s} h_{i}
$$

Case II $q=k, z=h$

$$
\left\{x_{1}, \ldots, x_{0}\right\} \text { is the set }\left\{h_{2}, \ldots, h_{k}, h 2^{n}\right\}
$$

in ascending order, and

$$
y=\frac{1}{h} g_{0} \prod_{i=k+1}^{s} h_{i}
$$

Case III $q=k, z=h_{0}$

$$
\left\{x_{1}, \ldots, x_{q}\right\} \text { is the set }\left\{h_{1} t_{1}, h_{2}, \ldots, h_{k}\right\}
$$


in ascending order, and

$$
\begin{aligned}
y & =g_{0} 2^{n} \prod_{i=k+1}^{s+m} h_{i} & \text { if } t_{1}=1 \\
& =g_{0} \prod_{i=k+1}^{s+m} h_{i} & \text { if } t_{1}=2^{n} .
\end{aligned}
$$

In all cases we get that $x_{1}, \ldots, x_{q}, y, z$ are integers such that

$$
\begin{array}{ll}
g=3 y \prod_{i=1}^{q} x_{i} & \\
\left(x_{i}, x_{j}\right)=1 & \text { for } 1 \leq i<j \leq q \\
(3, y)=\left(x_{i}, y\right)=1 & \text { for } 1 \leq i \leq q
\end{array}
$$

If $x_{i} \neq 1(\bmod 3)$ then $x_{i}=h 2^{n}$ in cases I or II. Thus $x_{i}>4 h \geq 20$.

\section{Therefore}

$$
x_{i} \equiv 1(\bmod 3) \text { or } x_{i}>20 \text { for } 1 \leq i \leq q .
$$

The inequalities (7), (8) and (9) become

$$
\frac{y \prod_{i=1}^{q} x_{i}}{15} \leq \frac{1}{2}\left(\frac{1}{3}-\frac{1}{z}\right) y \prod_{i=1}^{q} x_{i} \leq \sum_{i=1}^{q} x_{i}
$$

Lemma 10. $q \leq 2$. If $q=2$ then $y=1$.

Proof. If $x_{1}>4$ then by (13) $x_{1} \geq 7$. Hence (14) yields that

$$
7^{q-1} x_{q} \leq 15 \sum_{i=1}^{q} x_{i} \leq 15 q x_{q} .
$$

Thus $7^{q-1} \leq 15 q$ and so $q \leq 2$ in this case. If $x_{1} \leq 4$ then $x_{1}=4$ and

$$
4^{q-1} x_{q} \leq 15 \sum_{i=1}^{q} x_{i} \leq 15 q x_{q}
$$

Thus $4^{q-1} \leq 15 q$ and $q \leq 3$. Hence $q=3$ and by (14)

$$
4 x_{2} x_{3}<15\left(x_{1}+x_{2}+x_{3}\right)<45 x_{3} .
$$

Hence $x_{2}<12$. Thus by (11) and (12) $x_{2}=7$ and so $28 x_{3}<15\left(4+7+x_{5}\right)$ or $13 x_{3}<165$. Hence $x_{3}<13$ contrary to $7<x_{3}$, (11) and (12). Thus $q \leq 2$.

Suppose that $q=2$. Then (14) yields that $y x_{1} x_{2} \leq 15\left(x_{1}+x_{2}\right)$. If $y \geq 5$ this implies that $x_{1} x_{2} \leq 3\left(x_{1}+x_{2}\right)<6 x_{2}$. Thus $x_{1}=4$ and $4 x_{2} \leq 12+3 x_{2}$.. Hence $x_{2}=7$. Therefore $28 y \leq 15(11)=165$ and $y<6$. Therefore $y=5$ and by (10) $g=3 \cdot 4 \cdot 5 \cdot 7=420$. This is impossible since there is no simple group of order 
420. Thus $y<5$. If $y \neq 1$, then $y=2$ or $y=4$. If $y=2$, then $x_{1} x_{2}$ is odd and by (10) 4 does not divide $g$ contrary to the simplicity of $G$. Thus $y=4$. Hence $x_{1} x_{2}$ is odd and so $x_{1} \geq 7$. If $x_{1}>7$ then $x_{1} \geq 13$ and $52 x_{2} \leq 4 x_{1} x_{2} \leq 15\left(x_{1}+x_{2}\right)<30 x_{2}$ which is not the case. If $x_{1}=7$ then $28 x_{2} \leq 15\left(7+x_{2}\right)$ or $13 x_{2}<15.7$. Hence $x_{2}<13$ which is not the case. The lemma is proved in all cases.

LemMa 11. In case I or case II

$$
\frac{11}{75} y \prod_{i=1}^{q} x_{1} \leq \sum_{i=1}^{q} x_{1}
$$

Proof. $H_{s+1}$ admits $\Im_{3}$ as a group of automorphisms, thus $H_{s+1}$ is not cyclic. Hence $z=h=\left|H_{s+1}\right| \geq 25$ and the result follows from (14).

Lemma 12. $q=2, y=1$.

Proof. Suppose that $q=1$. Assume first that we have case I or II. Then Lemma 11 implies that $y<7$. Furthermore $|C(u)|=x_{1}$ and $[G: C(u)]=3 y$. Thus $y \neq 1$ and so by (12) $y=5$. Hence in case I (6) becomes

$$
\frac{11}{75} \cdot 5 \cdot 2^{n} h \leq h t_{s+1} \leq h 2^{n-1},
$$

or $\frac{22}{15} \leq 1$ which is not the case. In case II $\left|N\left(H_{1}\right)\right|=3 x_{1}$ and so $\left[G: N\left(H_{1}\right)\right]$ $=5$, thus $G$ is isomorphic to a subgroup of $\Im_{5}$. Hence $G$ is isomorphic to $\mathfrak{A}_{5}$ contrary to assumption.

Assume now that $q=1$ and we are in case III. Then (14) implies that $y \leq 15$. Since $G$ is simple $4 \mid g$. Thus by (10) and (12) either $y$ is odd or $4 \mid y$. Hence $y=4,8,5,7,11$ or 13 and $g=3 x_{1} y$. If $x_{1}$ is even then $x_{1}|| C(u) \mid$ and $x_{1} \neq|\boldsymbol{C}(\boldsymbol{u})|$. Since in this case $y=5,7,11$ or 13 , it is a prime. Hence $|\boldsymbol{C}(\boldsymbol{u})|$ $=x_{1} y$ and $[G: C(u)]=3$ which is impossible. If $x_{1}$ is odd then $x_{1} \equiv 1(\bmod 3)$, $y=4$ or 8 and $\left[G: N\left(H_{\mathrm{i}}\right)\right]=y$. Thus $y=8$ and $G$ is isomorphic to subgroup of $\Im_{8}$. As $H_{1}$ is nilpotent the Sylow theorems imply that the only prime dividing $x_{1}$ is 7 . As 49 does not divide 8 ! this implies that $x_{1}=7$. Hence $g=3 \cdot 7 \cdot 8$ and $G$ is isomorphic to $\operatorname{PSL}(2,7)$ contrary to assumption.

Hence $q=2$ and by Lemma $10 y=1$.

The proof of the main Theorem will now be completed.

By Lemma $12 g=3 x_{1} x_{2}$. In case I or II $H_{s+1}$ is not cyclic. Thus $z=h=$ $\left|H_{s+1}\right| \geq 25$. By (14) we get that 


$$
\frac{11}{75} x_{1} x_{2} \leq x_{1}+x_{2}<2 x_{2}
$$

Hence $x_{1}<14$. Thus $x_{1}$ is odd and $x_{1} \equiv 1(\bmod 3)$. This implies that $x_{1}=7$ or $x_{1}=13$. If $x_{1}=13$ then $\frac{11 \cdot 13}{75} x_{2} \leq 13+x_{2}$ or $25 \leq h \leq x_{2} \leq \frac{75}{68} \cdot 13$ which is not the case. Suppose that $x_{1}=7$. In case I (7) implies that

$$
2^{n} h<\frac{11}{75} 7 \cdot 2^{n} h \leq 7+2^{n-1} h .
$$

Hence $25<2^{n-1} h \leq 7$ which is not the case. In case II (6) implies that $2^{n} h<$ $\frac{11}{75} 7 \cdot 2^{n} h \leq 2^{n}+7+h t_{s+1} \leq 2^{n}+7+2^{n-1} h$. Hence $25 \leq 2^{n-1} h \leq 2^{n}+7$. So that $2^{n}>7$ and $2^{n-1} \cdot 25 \leq 2^{n-1} h<2^{n+1}$ which is not the case.

Assume now that we have Case III. Then $x_{1} \equiv x_{2} \equiv 1(\bmod 3)$, and by (14) $\frac{x_{1} x_{2}}{15} \leq x_{1}+x_{2}<2 x_{2}$. Hence $x_{1}<30$. If $x_{1}$ is even then $x_{1} \equiv 0(\bmod 4)$. If $x_{1}$ is a prime then $|C(u)|=x_{1} x_{2}$ and $[G: C(u)]=3$ which is not the case. Thus $x_{1}=4,16,25,28$. $\left[G: N\left(H_{2}\right)\right]=x_{1}$, thus $x_{1} \neq 4$. If $x_{1}=28$ then (14) yields that $\frac{28}{15} x_{2} \leq 28+x_{2}$ or $x_{2} \leq \frac{28 \cdot 15}{13}<33$. Hence $x_{2}=31$ is a prime. Thus $|C(u)|=x_{1} x_{2}$ and $[G: C(u)]=3$ which is not the case. If $x_{1}=25$ then $\frac{25}{15} x_{2} \leq 25+x_{2}$ or $x_{2} \leq$ $\frac{3}{2} 25<38$. Since $x_{2} \equiv 0(\bmod 4), x_{2}=28$. The Sylow theorems now imply that some divisor $d$ of 25 satisfies $d \equiv 1(\bmod 7)$ which is not the case. Assume finally that $x_{1}=16$. If $P$ is a Sylow subgroup of $H_{2}$ for some prime $p$ then $|N(P)|=3 x_{2}$ as $N\left(H_{2}\right)$ is a maximal solvable subgroup of $G$. Thus $16 \equiv 1(\bmod$ p). Hence $p=5$. Since $x_{2} \equiv 1(\bmod 3), x_{2}=5^{2 a}$ for some integer $a$. By (14) $\frac{16}{15} x_{2} \leq 16+x_{2}$ or $x_{2} \leq 240$. Thus $x_{2}=25$ and $g=3 \cdot 16 \cdot 25=1200$. There is no simple group of order 1200 .

This final contradiction establishes the main theorem of the paper.

\section{REFERENCES}

[1] R. Brauer and K. A. Fowler, On groups of even order, Ann. of Math. vol. 62 (1955), pp. 565-583.

[2] W. Burnside, Theory of Groups of Finite Order, Cambridge (1911).

[ 3 ] H. M. S. Coxeter and W. O. J. Moser, Generators and Relations for Discrete Groups, Ergebnisse, vol. 14 (1957).

[ 4 ] M. Suzuki, A new type of simple groups of finite order, Proc. Nat. Acad. Sci. vol. 46 (1960), pp. 868-870. 
[ 5 ] M. Suzuki, Finite groups with nilpotent centralizers, Trans. Amer. Math. Soc., vol. 99 (1961), pp. $425-470$.

Cornell University

University of Chicago and Harvard University 